\title{
FIXED POINTS OF SURFACE HOMEOMORPHISMS
}

\author{
BY BOJU JIANG (PO-CHU CHIANG)
}

For a self-map $f$ of a compact connected polyhedron $X$, the Nielsen number $N(f)$ is defined to be the number of essential fixed point classes (see [1] for an introduction to Nielsen fixed point theory). It is a classical theorem of Wecken [7] that $N(f)$ is a lower bound of the number of fixed points for all maps homotopic to $f$, and that if $X$ is a manifold of dimension $\geqslant 3$ this lower bound is always realizable (see also [1]). It is now known [3] that $N(f)$ is realizable if $X$ has no local cut points and $X$ is not a surface. The realizability problem for maps on surfaces is still open.

Historically, the theory of fixed point classes originated with Nielsen's study [5] of surface homeomorphisms. The purpose of this work is to examine the realizability of the Nielsen number of a surface homeomorphism as the least number of fixed points in an isotopy class. The same problem for higher-dimensional manifolds is open.

MAIN THEOREM. Let $M^{2}$ be a compact surface, closed or with boundary. Let $\varphi: M^{2} \rightarrow M^{2}$ be a homeomorphism. Then $\varphi$ is isotopic (through embeddings) to an embedding which has $N(\varphi)$ fixed points. If, in addition, no boundary component of $M^{2}$ is mapped onto itself by $\varphi$ in an orientation-reversing manner, then $\varphi$ is isotopic (through homeomorphisms) to a homeomorphism having $N(\varphi)$ fixed points.

The reason why we have to be contented with embeddings instead of homeomorphisms in the presence of an orientation-reversed invariant boundary component has already been explained in [4].

Our proof is based on Thurston's theory of surfaces $[6,2]$ which enables us to visualize a representative from each isotopy class.

For surfaces with $\chi\left(M^{2}\right) \geqslant 0$, the truth of the Theorem can be checked case by case. In the general case $\chi\left(M^{2}\right)<0$, Thurston [6] (see also [2]) tells us that every diffeomorphism $\varphi$ is isotopic to a "diffeomorphism" $\varphi$ ' which is either (1) an isometry with respect to a hyperbolic metric, or (2) a pseudo-Anosov "diffeomorphism", or (3) reducible in the sense that $M^{2}$ can be cut into simpler parts along an invariant set of disjoint simple closed curves, such that on each part, $\varphi^{\prime}$ is of type (1) or (2) in the complement of a collar around its boundary.

Received by the editors April 2, 1981.

1980 Mathematics Subject Classification. Primary 55 M20.

(c) 1981 American Mathematical Society 
The case (1) has already been discussed in [4]. The case (2) is related to Thurston's theorem [6, Theorem 6] that a pseudo-Anosov diffeomorphism has the minimum number of periodic points (for every period) in its isotopy class. However, Thurston's statement is true only for orientation-preserving homeomorphisms of orientable surfaces, although he did not say so explicitly. The reason is that a fixed point of an orientation-reversing pseudo-Anosov diffeomorphism which is an odd-pronged singularity (such an example is not hard to construct, say, on a closed surface of genus 2) has index 0 and is easily removable; boundary behavior can also cause trouble when the surface is bounded. Nevertheless, the truth of our Theorem in this pseudo-Anosov case can be shown by taking care of these pathologies.

The reducible case is much more complicated. To tackle it, we need the following key lemma which can be proved by a combinatorial group theory argument. (There is also a geometric proof of the lemma, due to A. Hatcher.)

Lemma. Let $M^{2}$ be a compact surface. Let $C=C_{1} \cup \cdots \cup C_{n}$ be a disjoint union of simple closed curves in Int $M^{2}$, none of which bounds a disc in $M^{2}$. Let $l: S^{1} \rightarrow M^{2}$ be a loop (homotopically) trivial in $M^{2}$. Assume that $l$ is transverse to $C$.

(i) If $l$ is in $M^{2}-C$, then $l$ is trivial in $M^{2}-C$.

(ii) If $l$ does cross $C$, then it must have at least two "loose segments".

Here by a "segment" we mean an $\operatorname{arc} A \subset S^{1}$ obtained by cutting $S^{1}$ along $l^{-1}(C)$; it is "loose" if $l \mid A$ can be deformed through $M^{2}-C$ into $C$ rel endpoints, i.e if $l \mid A$ can be extended to $h: D^{2} \rightarrow M^{2}$ such that $h^{-1}(C)=\overline{S^{1}-A}, D^{2}$ being the disc bounded by $S^{1}$.

This lemma helps us to analyze the structure of a fixed point class of $\varphi^{\prime}$. By isotoping $\varphi^{\prime}$ in the collars if necessary, a fixed point class can be made to consist of either a single point, an arc, a simple closed curve, or a connected subsurface possibly with some arcs sticking out of it. We also have detailed knowledge about the indices of the fixed point classes and the behavior of $\varphi^{\prime}$ near them. Then we can isotope $\varphi^{\prime}$ to change each fixed point class into a single point, or to remove it if its index is zero. This will complete the proof of the Theorem.

A by-product of our argument is

THEOREM. Let $M^{2}$ be a surface other than $S^{2}$. For any self-homeomorphism $\varphi: M^{2} \rightarrow M^{2}$, we have $N(\varphi) \geqslant$ the Lefschetz number $L(\varphi)$.

\section{REFERENCES} III., 1971.

1. R. Brown, The Lefschetz Fixed Point Theorem, Scott, Foresman and Co., Chicago,

2. A. Fathi, F. Laudenbach and V. Poénaru, Travaux de Thurston sur les surfaces, Séminaire Orsay, Astérisque 66-67 (1979). 
3. B. Jiang, On the least number of fixed points, Amer. J. Math. 102 (1980), 749763.

4. - Fixed point classes from a differential viewpoint, Proc. Workshop in Fixed Point Theory (Sherbrooke, 1980), Lecture Notes in Math., Springer-Verlag, Berlin and New York (to appear).

5. J. Nielsen, Untersuchungen zur Topologie des geschlossen zweiseitigen Flächen. I, Acta Math. 50 (1927), 189-358. (preprint).

6. W. Thurston, On the geometry and dynamics of diffeomorphisms of surfaces. I

7. F. Wecken, Fixpunktklassen. I, II, III, Math. Ann. 117 (1941), 659-671; 118 (1942), 216-234, 544-577.

DEPARTMENT OF MATHEMATICS, UNIVERSITY OF CALIFORNIA, LOS ANGELES, CALIFORNIA 90024

Current address: Department of Mathematics, Peking University, Peking, China 